\title{
A New Detection Method for Capacitive Micromachined Ultrasonic Transducers
}

\author{
A. Sanl Ergun, Associate Member, IEEE, Burak Temelkuran, \\ Ekmel Ozbay, Member, IEEE, and Abdullah Atalar, Senior Member, IEEE
}

\begin{abstract}
Capacitive micromachined ultrasonic transducers (cMUT) have become an alternative to piezoelectric transducers in the past few years. They consist of many small circular membranes that are connected in parallel. In this work, we report a new detection method for cMUTs. We model the membranes as capacitors and the interconnections between the membranes as inductors. This kind of $L C$ network is called an artificial transmission line. The vibrations of the membranes modulate the electrical length of the transmission line, which is proportional to the frequency of the signal through it. By measuring the electrical length of the artificial line at a high RF frequency (in the gigahertz range), the vibrations of the membranes can be detected in a very sensitive manner. For the devices we measured, we calculated the minimum detectable displacement to be in the order of $10^{-5} \AA / \sqrt{\mathrm{Hz}}$ with a possible improvement to $10^{-7} \AA / \sqrt{\mathrm{Hz}}$.
\end{abstract}

\section{INTRODUCTION}

$\mathrm{I}^{\mathrm{N}}$ THE PAST decade, silicon cMUTs started to become an alternative to piezoelectric transducers [1]-[5]. Using the standard silicon processes developed in the past 30 yr, along with micromachining technology, scientists developed reliable, small, and cheap transducers and transducer arrays with comparable or better performance both in air and immersion applications. Along with the capability of high density integration toward forming 1-D and 2-D arrays [6], [7], the main advantage of cMUTs turned out to be their good mechanical match to air without any matching layers [8], and over $100 \%$ bandwidth in water [9]-[11].

CMUTs consist of many circular membranes in parallel and are used for both generation and detection of ultrasound. The membrane is composed of a thin layer of dielectric (usually $\mathrm{SiN}_{x}$ ) coated with a metal electrode. A post layer supports the membrane over the substrate. The substrate is conducting and functions as a ground plane. The generation of ultrasound depends on the electrostatic attraction force between the top electrode and the substrate. When an alternating voltage with a large DC offset is applied to the electrode, the membrane vibrates, coupling acoustic power into the medium. For more detail on

Manuscript received February 25, 2000; accepted February 1, 2001. A. S. Ergun is with E. L. Ginzton Laboratory, Stanford University, Stanford, CA 94305-4085 (e-mail: sanli@piezo.stanford.edu).

B. Temelkuran is with MIT Physics Department, Boston, MA.

E. Ozbay is with Bilkent University Physics Department, 06533 Bilkent, Ankara, Turkey.

A. Atalar is with Bilkent University Electrical and Electronics Engineering Department, 06533 Bilkent, Ankara, Turkey. the analysis of the membrane structure and experimental results, the reader may refer to the previous work of Khuri-Yakub et al. [12]-[15].

The membrane that generates ultrasound is used for detecting it as well. The electrode and the substrate make up a simple parallel plate capacitor. The detection of ultrasound depends on the vibration of the membrane caused by an incident ultrasonic signal. The displacement of the membrane results in a capacitance change that is measured by monitoring the current under a constant bias voltage. The magnitude of the current resulting from $n$ parallel membranes can be expressed as

$$
I=2 \pi f_{1} V_{d c} n C \frac{\Delta x}{x_{0}}
$$

where $f_{1}$ is the ultrasound frequency, $V_{d c}$ is the bias voltage, $C$ is the capacitance of a single membrane, $x_{0}$ is the separation between the electrode and the substrate, and $\Delta x$ is the magnitude of the displacement. The resulting current is then amplified with a transimpedance amplifier.

To increase the displacement sensitivity of a cMUT as an ultrasonic detector, there are three parameters to optimize. We can increase the bias voltage, which is limited by the collapse voltage. We can decrease the gap between the membrane and the substrate. But, this is not as effective as it seems because decreasing the gap decreases the collapse voltage as well. Finally, we can increase the total capacitance by increasing the number of membranes $(n)$ and/or the area of the individual membrane.

In this paper, we demonstrate an alternative method to detect the displacement of a membrane in a more sensitive manner, which was introduced in a previous work [16], [17]. Similar to the conventional method, our method is also based on the membrane capacitance variation upon receiving an ultrasound signal. However, as a major difference, we pattern the top electrode covering the membrane in the form of a transmission line and transmit a high frequency signal (in the gigahertz range) through it instead of applying a DC bias voltage. The vibrations picked up by the membranes cause a phase modulation on the transmitted RF signal. We detect the membrane vibrations by demodulating the transmitted signal, which turns out to be a very sensitive way of detecting mechanical vibrations. 


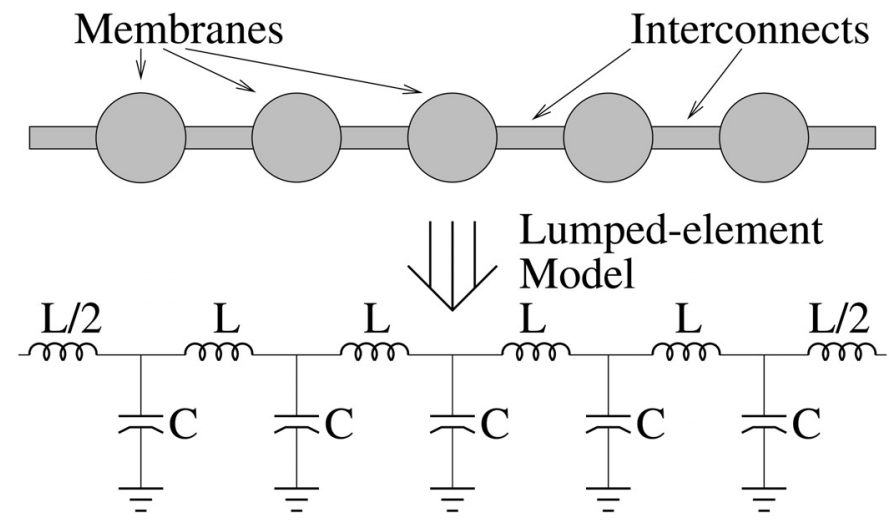

Fig. 1. The lumped element circuit model of a detector with five elements.

\section{The NeW Method}

The electrical model of a membrane is a simple parallel plate capacitor. The membranes are connected through interconnection lines. However small, these interconnection lines can be modeled as lumped inductors. If the membranes are integrated in a series manner, as shown in Fig. 1, then the resulting lumped-element circuit model is an $L C$ network, which may be called an artificial transmission line. Note that the acoustical port of the detector is omitted for the time being because the frequency of interest is much higher than the mechanical resonance frequency of the detector. From now on, we will call such signals RF signals.

An artificial transmission line has a Bragg frequency $\left(\omega_{\text {bragg }}=2 / \sqrt{L C}\right)$ at which there is total reflection. For frequencies well below the Bragg frequency, it has a characteristic impedance $Z_{a}$ and a propagation constant $\beta$ determined by the $L$ and $C$ values as shown in (2) and (3), where $f_{0}$ is the frequency:

$$
\begin{aligned}
Z_{a} & =\sqrt{\frac{L}{C}} \\
\beta & =2 \pi f_{0} \sqrt{L C} .
\end{aligned}
$$

The propagation constant $\beta$ has units of radians per section. Thus, the electrical length $(\Phi)$ of the artificial transmission line is

$$
\Phi=n \times \beta
$$

where $n$ is the number of sections. At low frequencies (in the low megahertz range), the electrical length of the artificial transmission line is negligible. Practically, all of the membranes are in parallel, and the detector is similar to a single lumped capacitor, as it is assumed in the generation and conventional detection of ultrasound. As the frequency increases, the electrical length of the artificial transmission line increases proportionally. Because the electrical length is not negligible anymore, the detector becomes a distributed element at these frequencies.

At the quiescent position $\left(x=x_{0}\right)$, the membranes have a definite capacitance, and the electrical length of the line

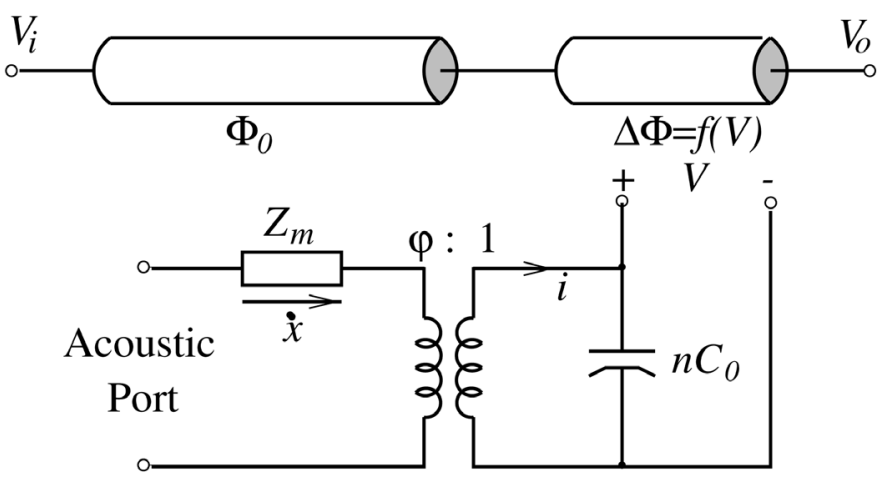

Fig. 2. The electrical model of the detector.

is fixed at $\Phi_{0}=\Phi\left(x_{0}\right)$. If a high frequency RF signal is applied from one of the ports, then the transmitted signal measured from the other port is a replica of the input with a phase shift that is equal to $\Phi_{0}$. When the membranes displace from their quiescent position with the effect of an external pressure, the electrical length of the line and the phase of the transmitted signal changes. In other words, the phase of the transmitted signal is modulated by the pressure on the membranes. For example, if an ultrasound signal at $2 \mathrm{MHz}$ is incident on the membranes, the phase of the transmitted RF signal is modulated at $2 \mathrm{MHz}$. In the new method, the external pressure is detected by phasedemodulating the transmitted signal.

\section{Mathematical Formulation}

We include the acoustical port of the detector in the model as shown in Fig. 2. The electrical side consists of two transmission lines. One of them has a fixed electrical length $\Phi_{0}$. The other one is a controlled phase-shifter whose length is controlled by the mechanical port. The mechanical port of the detector is modeled as usual, which is the Mason's equivalent [18].

The membrane is a parallel plate capacitor whose capacitance is $C_{p p}=A \epsilon_{0} / x$, where $A$ is the effective capacitor area and $x$ is the parallel plate separation. In addition, there are always fringing capacitances that add to the parallel plate capacitance and have very weak $x$ dependence. The interconnection lines between the membranes are modeled as inductors, which is mostly true for high impedance and short lines. However, there is always a small parasitic capacitance associated with each inductor. This capacitance also adds to the parallel plate capacitance. Defining the sum of the fringing and parasitic capacitances as $C_{f}$, the total capacitance $C$ at the membrane node is written as $C=A \epsilon_{0} / x+C_{f}$. Assuming an $n$-section artificial transmission line with identical membranes, the electrical length $\Phi$ can be written as $\Phi=n \cdot 2 \pi f_{0} \sqrt{L C}$.

At the quiescent position of the membrane, the total capacitance is denoted as $C_{0}=C\left(x_{0}\right)$, and the propagation constant is denoted as $\beta_{0}=\beta\left(x_{0}\right)$, such that $\Phi_{0}=n \beta_{0}$. The vibration of a membrane means that the parallel plate separation $x$ vibrates around the quiescent position $x_{0}$. As 
a result, the capacitance of the membrane and the electrical length of the artificial transmission line vibrate around their quiescent values. We can expand $\Phi$ around $x=x_{0}$ :

$$
\Phi(x(t))=\Phi_{0}+\Delta \Phi(\Delta x(t))
$$

where $\Delta x(t)=x(t)-x_{0}$. Then,

$$
\Delta \Phi(\Delta x(t))=-\frac{n \beta_{0}}{2 x_{0}} \frac{C_{p p 0}}{C_{0}} \Delta x(t)
$$

where $C_{p p 0}=C_{p p}\left(x_{0}\right)$. For a $\mathrm{RF}$ signal of $V_{i}=$ $V_{R F} \cos \left(2 \pi f_{0} t\right)$ input to the detector, the output is (assuming that the artificial line is matched to the source)

$$
V_{o}=V_{R F} \cos \left[2 \pi f_{0} t-\Phi_{0}-\Delta \Phi(\Delta x(t))\right] .
$$

Consequently, the output of the detector is a phasemodulated signal as discussed before.

In the well-known Mason's equivalent, the mechanical and electrical port of the detector are connected through a transformer. The transformer relates the velocity at the mechanical port to the current at the electrical port. Therefore, the transformer ratio denoted by $\varphi$ is expressed in Coulomb per meter. By defining the transformer ratio as

$$
\varphi=\frac{\Phi_{0} n C_{p p 0}}{2 x_{0}} V_{R F},
$$

where $V_{R F}$ is the RF signal amplitude, we can write $\Delta \Phi(\Delta x(t))$ as a controlled quantity as shown in (7):

$$
\Delta \Phi(\Delta x(t))=-\frac{\varphi}{n C_{0} V_{R F}} \Delta x(t) .
$$

The displacement of the membranes $(\Delta x(t))$ can be detected by measuring $\Delta \Phi(\Delta x(t))$ of the transmitted signal. One way to obtain $\Delta \Phi(x(t))$ is to use an interferometer, which is one of the basic methods for phase measurements. We first divide the input signal into two. One-half goes through a transmission line with fixed electrical length. The other half goes through the artificial transmission line made up of membranes. Then, the signals are summed again as shown in Fig. 3(a). The amplitude of the interferometer's output is a function of the electrical length difference between the two arms, which is modulated by the vibrations picked up by the membranes. The result is an amplitude-modulated signal at the output of the interferometer. The vibration amplitude of the membranes is measured by envelope detection.

Using a narrow-band phase modulation approximation $(\Delta \Phi(x(t)) \ll 1)$, we can write the output of the envelope detector as shown in (8):

$$
V_{\text {out }}=V_{R F}\left|\cos \left(\frac{\Phi_{d}}{2}\right)+\sin \left(\frac{\Phi_{d}}{2}\right) \frac{\Delta \Phi(x(t))}{2}\right| .
$$

The quiescent phase difference between the two arms $\Phi_{d}$ should be chosen such that $\sin \left[\Phi_{d} / 2\right]$ is close to but not equal to 1 to achieve the optimum sensitivity. Note that the choice of $\Phi_{d}=\pi$ causes distortion and should be avoided.

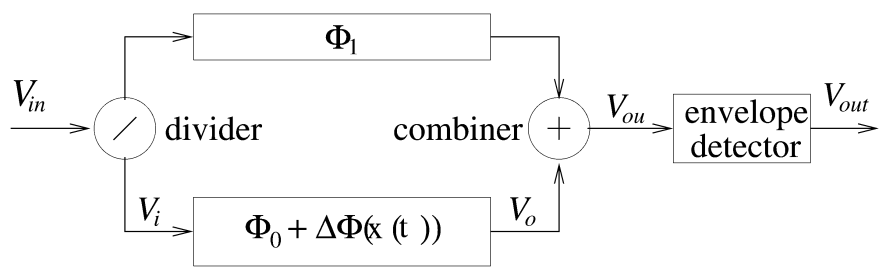

(a)

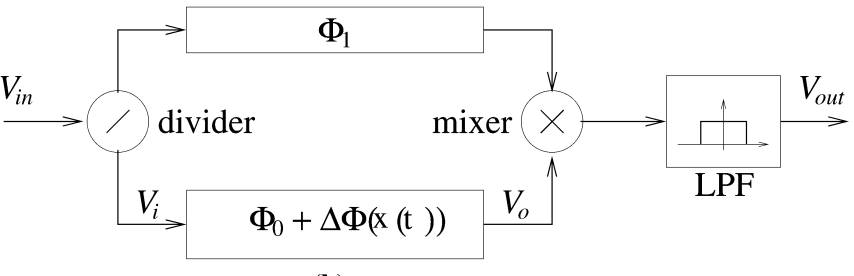

(b)

Fig. 3. The interferometric (a) and direct method (b) to measure the phase modulation.

Membrane vibrations can also be detected by directly mixing the phase-modulated signal with a reference signal and subsequent low-pass filtering as shown in Fig. 3(b). Using the narrow band phase-modulation approximation $(\Delta \Phi(x(t)) \ll 1)$ again, we can expand the cosine term in (5) and make necessary approximations to obtain

$$
\begin{aligned}
V_{o} & =V_{R F}\left[\cos \left(2 \pi f_{0} t-\Phi_{0}\right)\right. \\
& \left.+\sin \left(2 \pi f_{0} t-\Phi_{0}\right) \Delta \Phi(x(t))\right] .
\end{aligned}
$$

If we assume a sinusoidal vibration for the membranes such as $x(t)=x_{0}+\Delta x \sin \left(2 \pi f_{1} t\right)$, then the amplitude spectrum of the transmitted signal contains a main signal at the RF frequency $f_{0}$ and two sidebands at $f_{0} \pm f_{1}$. The sidebands have amplitudes determined by the vibration magnitude of the membranes. We measure the amplitude of the sidebands $\left(V_{\text {out }}\right)$ to obtain $\Delta x$, where

$$
V_{\text {out }}=V_{R F} \frac{n \beta_{0} C_{p p 0}}{4 x_{0} C_{0}} \Delta x .
$$

If we rewrite (10) in terms of the current, we obtain

$$
I_{\text {out }}=2 \pi f_{0} \frac{V_{R F}}{4} n C_{p p 0} \frac{\Delta x}{x_{0}},
$$

which looks similar to the output current in conventional detection. We can compare the new method with the conventional one by comparing (1) and (11). The DC bias voltage term (which is usually in tens of volts in range) in the conventional method is replaced by a few volts of $\mathrm{RF}$ amplitude term in our method. The reduction in voltage magnitude is compensated with the replacement of the vibration frequency $f_{1}$ by the RF frequency $f_{0}$. Considering a vibration frequency in the megahertz range for the membranes and an RF signal in the gigahertz range, a few orders of magnitude improvement in the sensitivity over the conventional method is possible. For applications that involve lower vibration frequencies (kilohertz range or lower), the improvement is even better. 


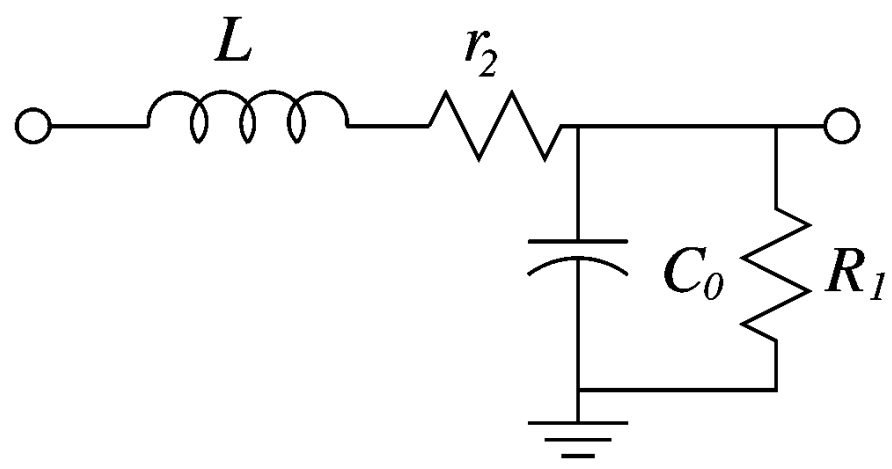

Fig. 4. A single section lossy artificial transmission line.

We define the sensitivity of the detector as the change in the output voltage for a unit displacement in the membrane positions:

$$
S=\left|\frac{\Delta V_{\text {out }}}{\Delta x}\right|
$$

Eqs. (8) and (10) imply that the sensitivity of the detector increases indefinitely with $n$. However, this is not the case. The loss of the artificial transmission line is determined by the attenuation constant $\alpha$, and it grows exponentially with $n$. This loss causes a reduction in the output, and the sensitivity of the direct detection is expressed as

$$
S=V_{R F} e^{-\alpha n} \frac{n \beta_{0}}{4 x_{0}} \frac{C_{p p 0}}{C_{0}} .
$$

This sensitivity expression contains a term that increases linearly with $n$ and a term that decreases exponentially with $n$. This means that there is an optimum number of sections $n_{\text {opt }}$ beyond which the output starts to decrease with increasing $n$. The optimum $n$ value is determined by taking the derivative of the sensitivity expression with respect to $n$, which gives $n_{\text {opt }}=1 / \alpha$. The maximum sensitivity obtained for $n=n_{\text {opt }}$ can be written as

$$
S_{\max }=V_{R F} \frac{\beta_{0}}{4 e \alpha x_{0}} \frac{C_{p p 0}}{C_{0}} .
$$

The sensitivity is proportional to the RF frequency, which is inherent in $\beta_{0}$. Therefore, we may expect to get as much sensitivity as we want if we go up in frequency. However, this is not the case because of the exponential increase in the RF losses.

The main loss mechanism in the artificial transmission line we are concerned with is the resistance of the interconnection lines between the membranes. This ohmic loss can be modeled as a resistance $r_{2}$ in series with the inductors as shown in Fig. 4. Another loss mechanism is the shunt losses associated with the finite resistivity and the loss tangent of the substrate. This loss can be modeled as a resistor $R_{1}$ in parallel with the capacitors. The attenuation constant for a lossy artificial transmission line is derived in [19], which is

$$
\alpha=\frac{r_{2}}{2 Z_{a}}+\frac{Z_{a}}{2 R_{1}} .
$$

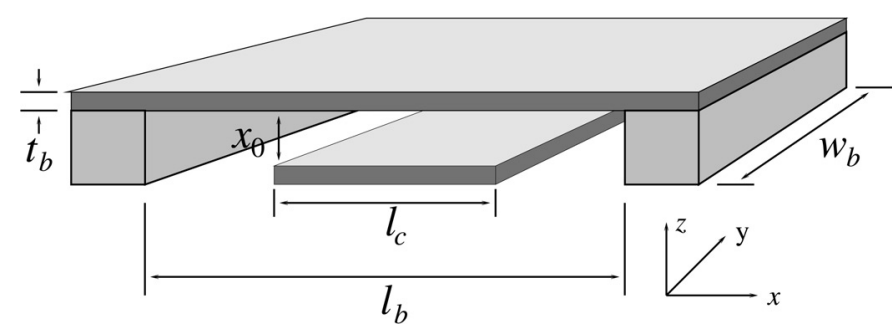

Fig. 5. A simplified schematic of an air-bridge.

For semi-insulating substrates, the ohmic loss is the dominant one so that the second term in (15) can be omitted.

\section{Device Design and Fabrication}

Although we have mentioned only membranes until now, the new method can also be applied to similar structures. The main idea is to integrate the microelectromechanical capacitive device in the form of an artificial transmission line and use a high frequency RF signal to sense the displacement of the device. In this work, we used air-bridges as ultrasonic transducers instead of membranes for faster proof of the method because of its ease of fabrication. The way to incorporate this method in regular membrane structures is a little bit more tricky and will be discussed later.

The air-bridge structure, which is a new type of capacitive ultrasonic transducer, is very similar to a rectangular membrane. The only difference is that an air-bridge has two free edges as shown in Fig. 5, whereas a rectangular membrane is clamped on all edges. For this reason, airbridge transducers are not suitable for immersion applications. The gap cannot be isolated from the medium, and water inside the gap kills the membrane vibrations. However, the free edges are advantageous in air applications. It provides higher average displacement and larger aperture for the air inside the gap to go in and out. This decreases the streaming air resistance and the squeeze film damping. There are a few problems though with the air-bridge structure used here. It is not repeatable because of the bridge material (aluminum) whose residual stress is not controllable. The other problem is the durability. In short, the problems of the air-bridge transducer are associated with the membrane material and the fabrication process. With a couple of changes in the process and switching to a dielectric bridge material metalized on top, the air-bridge transducer can be improved considerably. However, this is not the scope of this work.

The air-bridge transducers we fabricated basically consist of a co-planar waveguide (CPW) line, which is periodically loaded with air-bridges. CPW is a planar transmission media in which the signal line is located between two ground planes. The air-bridges connect the two ground planes and, therefore, pass over the signal line. This creates a capacitance between the signal line and the ground, which makes up the capacitive transducer (Fig. 6). We 


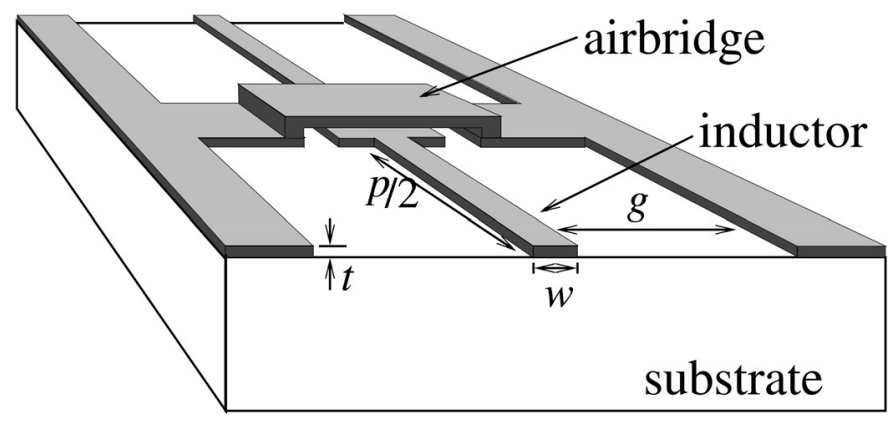

Fig. 6. A section of an artificial transmission line with an air-bridge.
TABLE I

Geometrical Parameters of a Unit Section.

\begin{tabular}{ccc}
\hline Symbol & Description & Value $(\mu \mathrm{m})$ \\
\hline$p$ & Inductor length & 60 \\
$w$ & Inductor width & 10 \\
$g$ & CPW gap & 24 \\
$l_{c}$ & Capacitor length & 30 \\
$w_{c}$ & Capacitor width & 40 \\
$t$ & Metalization thickness & 1 \\
$l_{b}$ & Bridge length & 80 \\
$x_{0}$ & Bridge height & 0.35 \\
$t_{b}$ & Bridge thickness & 1.2 \\
\hline
\end{tabular}

$2695\left(\mathrm{~kg} / \mathrm{m}^{3}\right)$. Inserting these values in (16), the first resonance frequency of the $\mathrm{Al}$ air-bridge, $\nu_{1}$, simplifies to

$$
\nu_{1}=4970 \frac{t_{b}}{l_{b}^{2}} \quad(\mathrm{MHz})
$$

where $t_{b}$ and $l_{b}$ are in units of microns. In this work, we chose the bridge thickness to be $1.2 \mu \mathrm{m}$. Our design goal for the resonance frequency is something above $1 \mathrm{MHz}$. Among various designs we fabricated, we will demonstrate results on two particular ones. They have bridge lengths of 80 and $70 \mu \mathrm{m}$, which puts the first mechanical resonance frequency at 0.93 and $1.2 \mathrm{MHz}$, respectively.

The mechanical bandwidth of the air-bridge is determined by the structural and air-streaming losses. Although these losses cannot be calculated easily and accurately, finite element modeling and simulations can predict the losses. However, both analytic calculations and finite element simulations fall out of the scope of this work and, therefore, are skipped.

For the electrical design of the detector, we have two objectives. One of them is to make the propagation constant $\beta$ as large as possible. The other one is to keep the losses as small as possible. All of these parameters can be calculated analytically, which provides us with a good starting point. The calculations do not include the parasitic capacitances, inductances, or second-order losses. Therefore, it needs electromagnetic simulations to fine tune the design and predict the performance of the detectors more accurately. The objective of electromagnetic simulations is to determine the lumped element model of a unit section (Fig. 4) from which we are able to calculate the propagation and attenuation constants and the sensitivity of the detector.

Table I summarizes the geometrical parameters of one of our designs. We use a high resistivity GaAs substrate with a dielectric constant of 12.9. The metal we use is aluminum, which has a resistivity of $2.67 \mu \Omega \cdot \mathrm{cm}$. We used the EM simulator of Sonnet Software, which also extracts the lumped element model of the structure, and calculate $L, C, r_{2}$, and $R_{1}$ values. The results are shown in Tables II and III as a function of frequency for $V_{R F}=1 \mathrm{~V}$. We removed the shunt loss resistor $R_{1}$ from the tables, because it is quite large and has no effect on the attenuation constant. This implies that the loss is totally dominated trode without collapsing and still give reasonable resonance frequencies: $Q_{A l}=6.3 \times 10^{10}\left(\mathrm{~N} / \mathrm{m}^{2}\right)$ and $\rho_{A l}=$ $\beta_{n}$ are the constants with the values $\beta_{1}=1.5056, \beta_{2}=$ 2.4997 , and $\beta_{n}=n+0.5$ for $n>2$.

Although dielectrics are far superior to metals in terms of mechanical properties, aluminum has reasonably good mechanical properties too. For practical bridge dimensions, it can support the bridge above the bottom elec- 
TABLE II

Extracted Lumped Element Values of the Unit Section of Table I.

\begin{tabular}{ccccc}
\hline $\begin{array}{c}f_{0} \\
(\mathrm{GHz})\end{array}$ & $\begin{array}{c}L \\
(\mathrm{pH})\end{array}$ & $\begin{array}{c}C \\
(\mathrm{fF})\end{array}$ & $\begin{array}{c}r_{2} \\
(\Omega)\end{array}$ & $\begin{array}{c}Z_{a} \\
(\Omega)\end{array}$ \\
\hline 1 & 88.5 & 48.6 & 0.188 & 42.7 \\
2 & 65.6 & 48.6 & 0.273 & 36.7 \\
5 & 57.5 & 48.6 & 0.347 & 34.4 \\
10 & 55.0 & 48.8 & 0.401 & 33.6 \\
20 & 53.3 & 49.5 & 0.479 & 32.8 \\
\hline
\end{tabular}

TABLE III

Calculated Values Using the Lumped Element Model of Table II.

\begin{tabular}{ccccc}
\hline $\begin{array}{c}f_{0} \\
(\mathrm{GHz})\end{array}$ & $\begin{array}{c}S_{u} \\
(\mathrm{~V} / \mu \mathrm{m})\end{array}$ & $n_{0}[\mathrm{opt}]$ & $\begin{array}{c}\Phi_{0} \\
(\mathrm{rad})\end{array}$ & $\begin{array}{c}S_{\max } \\
(\mathrm{V} / \mu \mathrm{m})\end{array}$ \\
\hline 1 & 0.0067 & 450 & 5.9 & 1.1 \\
2 & 0.0114 & 268 & 6.0 & 1.1 \\
5 & 0.0268 & 198 & 10 & 2.0 \\
10 & 0.0522 & 167 & 17 & 3.2 \\
20 & 0.102 & 137 & 28 & 5.2 \\
\hline
\end{tabular}

by the conductor losses. Notice that we have included $S_{u}$ in Table III, which corresponds to the sensitivity of a unit section. For small devices with few sections, the sensitivity can be calculated by multiplying $S_{u}$ with the number of sections.

Using the $L$ and $C$ values, it is possible to calculate the Bragg frequency of the line in Table I, which turns out to be around $1 \mathrm{THz}$. The frequencies we are interested in are well below the Bragg frequency, which means that the $L C$ model we assume in the calculations is valid.

As predicted before, we see that the loss increases with frequency. Consequently, the optimum number of sections $\left(n_{0}[\mathrm{opt}]\right)$ in Table III decreases with increasing frequency. The sensitivity plot shown in Fig. 7, as a function of $n$ and $f_{0}$, shows the detector performance more clearly. For con-

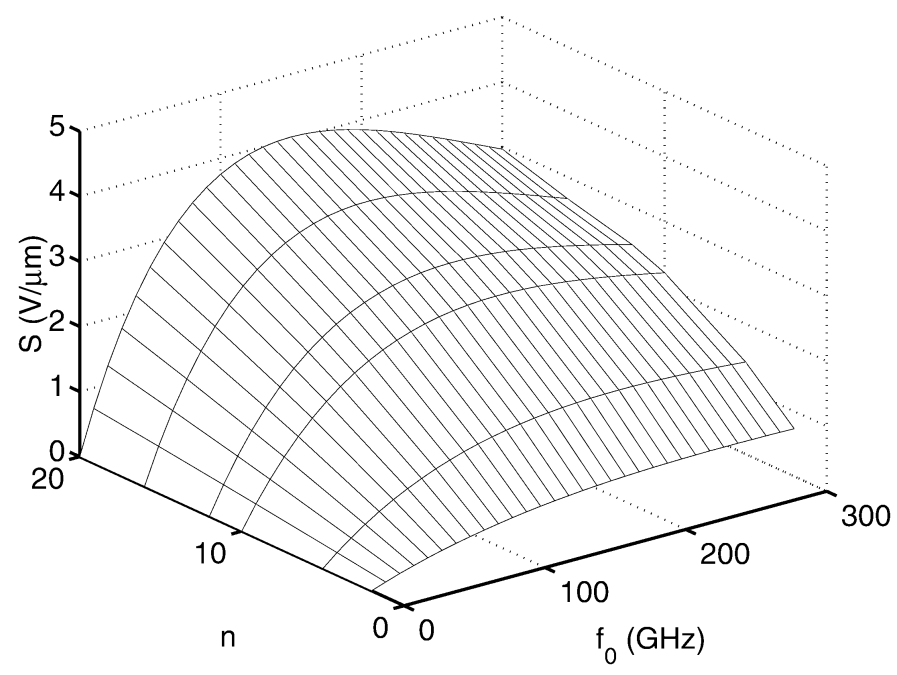

Fig. 7. Sensitivity as a function of $n$ and $f_{0}$. stant frequency, there is an optimum number of sections that maximizes the sensitivity. The plot also tells that for this particular design, we may get better sensitivity figures if we go up in frequency.

The design shown here is actually not an optimum design. The sensitivity can be further increased. The main concern is to increase the propagation constant (3) while keeping the losses low for a fixed unit cell. This can be accomplished by optimizing the geometrical parameters shown in Table I. Obviously, some of these parameters conflict with each other, such as inductor length and capacitor width, and we have to find an optimum point. EM simulations show that the optimum point is the capacitor covering between 60 to $80 \%$ of the unit cell. We may also decrease the inductor width to increase the inductance, but it also increases the losses. Although this parameter does not have much effect on the overall sensitivity, EM simulations show that an inductor width of $20 \mu \mathrm{m}$ is the best choice. The capacitor length and the gap height are the two parameters that do not conflict with any other. We can increase the capacitor length as much as the unit cell allows and decrease the gap height as much as the process allows. They both result in a good increase in sensitivity. One last important parameter is the metalization, which should be as thick as practical. It decreases the losses and makes longer lines possible.

All of the simulations suggest that we make the capacitor larger to get more sensitivity. There is one downside of it though, which is the characteristic impedance of the line. It drops to teens for the optimum geometry, which creates a great mismatch to a $50-\Omega$ system. This may suggest big reflections and loss of RF signal, but it is not the case. Because this detector is supposed to work at a single $\mathrm{RF}$ frequency, matching it to $50 \Omega$ is fairly easy. Another possibility is to design the whole electronics to match the artificial transmission line.

It is important to note that, for substrates with lower resistivity, the substrate losses dominate the conductor losses, which degrades the sensitivity considerably. Recall that, in usual cMUTs, SiN membranes are fabricated on heavily doped silicon, which is used as a ground plane. This occurs to be a problem in applying this new method to membrane devices. However, this can be solved by using a semi-insulating or insulating substrate and defining the ground plane with a separate metalization.

The fabrication of the transducers is rather simple. We used a standard air-bridge process. A simplified process diagram is shown in Fig. 8. The first step is a metalization step, which defines the CPW line. The next step is the sacrificial layer growth, which will be removed in the end. We used hard-baked photoresist as a sacrificial layer. After spinning $1.5 \mu \mathrm{m}$ of positive photoresist, we pattern it using conventional lithography. Then, we hard bake it at $140^{\circ} \mathrm{C}$ for $30 \mathrm{~min}$. Finally, we thin it down to the desired thickness with plasma etching. The third step is the bridge metalization step. At last, the device is soaked in acetone for sacrificial layer removal and cleaned with reactive ion etching (RIE). 
$\Gamma$

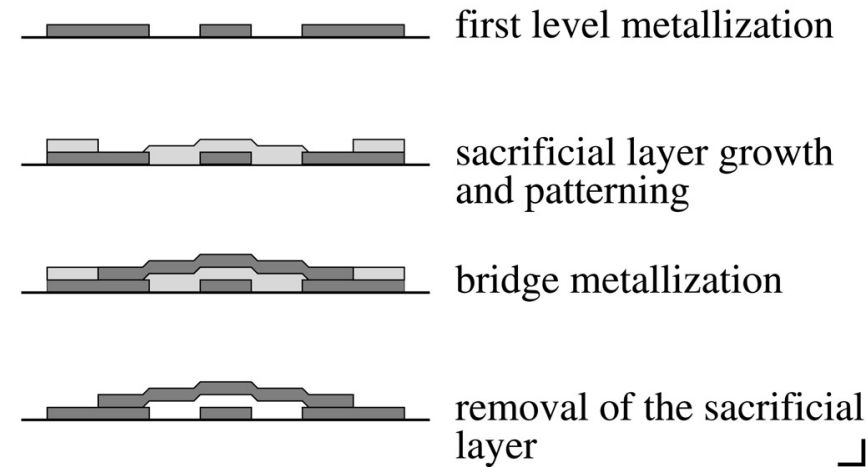

Fig. 8. Simplified diagram of an air-bridge process.

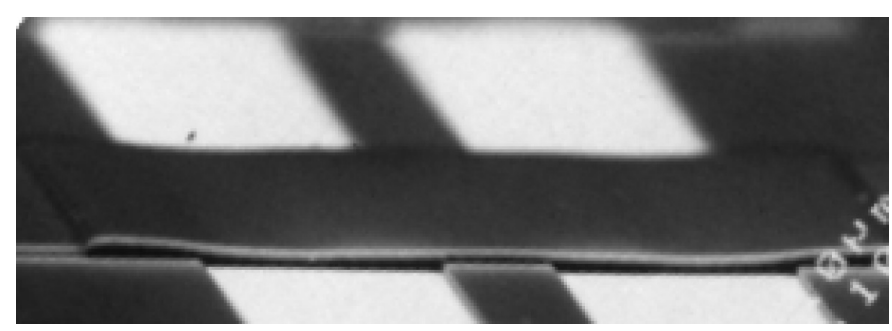

Fig. 9. SEM photograph of an artificial transmission line section with an air-bridge.

In all of the metalization steps that are done by lift off, image reversal technique is used instead of the conventional lithography. Image reversal makes the lift-off process much easier, especially for thick metalizations. In addition, it provides clean edges without any overshoots, which is crucial for the air-bridges. The SEM photograph of a finished device in Fig. 9 shows a unit section. The geometrical parameters of this device, such as metalization thickness, bridge thickness, and gap height, are those listed in Table I.

\section{Experimental Results}

We have performed five basic experiments on the fabricated devices. First, we measured the lumped element values that were predicted by the electromagnetic simulations to see the accuracy of our model. Then, we measured the static mechanical behavior of the air-bridges, which we compared with the predicted sensitivity. The third experiment is the excitation experiment. It gives us the dynamic mechanical response of the air-bridges from which we can extract the Mason's equivalent circuit for the mechanical port. The other two experiments are transmit-receive and pulse-echo experiments.

It is possible to extract the lumped element values of an artificial transmission line that are shown in Fig. 4 from the measured S-parameters. We first convert the S-parameters into ABCD parameters. Then, we can extract the characteristic impedance and the propagation constant of the artificial transmission line from the ABCD parameters from which we can calculate the lumped element values [21].

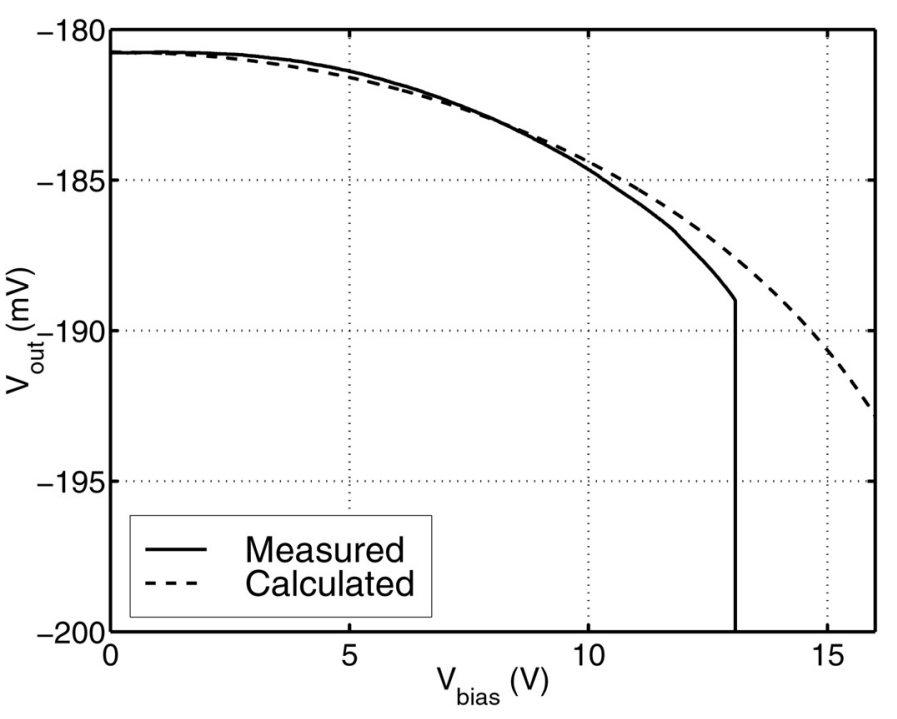

Fig. 10. The result of the interferometric detection experiment performed on electrostatically deflected bridges.

When we compare the measurement and simulation results, we see that $C_{0}, r_{2}$, and $R_{1}$ are predicted very well with the electromagnetic simulation. The measured value of $L$ is slightly smaller than the predicted value. This difference is about $10 \%$ and decreases for devices that have wider bridges. The effect of this difference on our calculations is not significant at all. Then, we conclude that we can use the lumped model extracted by the electromagnetic simulations with good accuracy.

When we apply a DC bias between the signal and the ground planes of the CPW, there is an electrostatic attraction force between the two plates of the capacitor. Being the top plate of the capacitor, the bridge deflects down. The amount of deflection can be calculated as described in [22], [23], where the spring constant of the bridges is measured by surface texture analysis. Thus, using the new method with interferometric detection, we can measure the deflection of the bridges and compare it with the calculated values.

We apply the DC bias through a bias- $T$. The RF signal is at $2 \mathrm{GHz}$ with $10-\mathrm{dBm}\left(V_{R F}=1 \mathrm{~V}\right)$ power. The $\mathrm{DC}$ bias voltage is swept from 0 to the collapse voltage with steps of $10 \mathrm{mV}$. The output is passed through a DC amplifier of gain 10 and is measured with a multimeter through HPIB interface. The measurement result is plotted in Fig. 10 together with the calculation result. The device contains 44 sections, where the geometrical parameters are those listed in Table I. The calculation result assumes that the bridge height is uniform throughout the device with a value of $x_{0}=0.35 \mu \mathrm{m}$. The spring constant is measured to be $k=275 \mathrm{~N} / \mathrm{m}$ as found by surface texture analysis.

We see in Fig. 10 that the measured result is in good agreement with the calculated one. The slight difference is because of the bridge height distribution. The bridge height is not uniform over the 44 section device as assumed in the calculations. Because of the fabrication process, it has some variation. This is already evident from the lower 


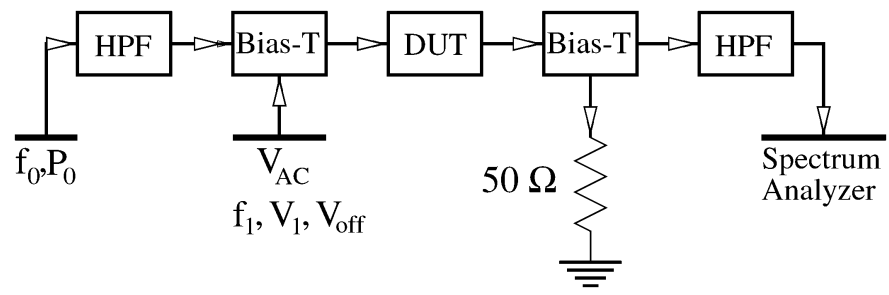

Fig. 11. Excitation experiment setup. $V_{A C}$ is used to vibrate the bridges through a bias $\mathrm{T}$. The RF source feeds the artificial transmission line, and the spectrum analyzer monitors the transmitted signal.

collapse voltage of the measured data. Detailed discussion on this subject can be found in [19].

Our third experiment is the excitation experiment in which we use the transducer as a transmitter. We apply an AC signal on top of the DC bias voltage to vibrate the bridges and vary the frequency to see the frequency response. At the same time, we apply the high frequency $\mathrm{RF}$ signal through a bias- $\mathrm{T}$ to measure the vibration amplitude of the bridges.

The excitation experiment setup is shown in Fig. 11. This setup is actually a simplified form of the direct detection method in Fig. 3(b). The splitting of the signal into a reference arm, mixing with the modulated signal and subsequent low-pass filtering, are all done by the spectrum analyzer. On the spectrum analyzer display, we see the main signal at $f_{0}$ and the sidebands at $f_{0} \pm f_{1}$. We measure the sideband peaks, convert them to voltage, and plot. The numbers we obtain are actually equal to the numbers we would get from a direct detection circuitry with ideal components. The high-pass filters are added to prevent the low-frequency excitation loading the RF source and the spectrum analyzer.

We perform this excitation experiment on the same type of device for which simulation results are shown. The AC voltage is $5 \mathrm{~V}$, peak to peak. The $\mathrm{RF}$ is $2 \mathrm{GHz}$ with an amplitude of $1 \mathrm{~V}$. According to (16), we expect the resonance frequency to be $0.93 \mathrm{MHz}$. Fig. 12 shows the experimental results of devices with 8,22 , and 44 sections. The measured resonance frequency is $\sim 1.39 \mathrm{MHz}$, which is substantially higher than expected. The reason for this difference is the internal residual stress, which is ignored in (16). Another source of this difference is probably the squeezed film damping. Using the measured data, we find that the vibration amplitude is $6 \AA / \mathrm{V}$ for this device at its resonance frequency. This figure is considerably lower than the typical silicon nitride membranes [1]. The reason is the high mechanical losses, which are evident from the low quality factor of the air-bridge transducers (around 4 for this particular device).

Fig. 13 shows the results of the excitation experiments done on another device with 22 sections. The bridge length, width, and height of this device are 70, 80, and $0.4 \mu \mathrm{m}$, respectively. The capacitor width on the other hand is $26 \mu \mathrm{m}$ for this device. The rest of the parameters are the same as the previous one. The AC voltage, $\mathrm{RF}$, and

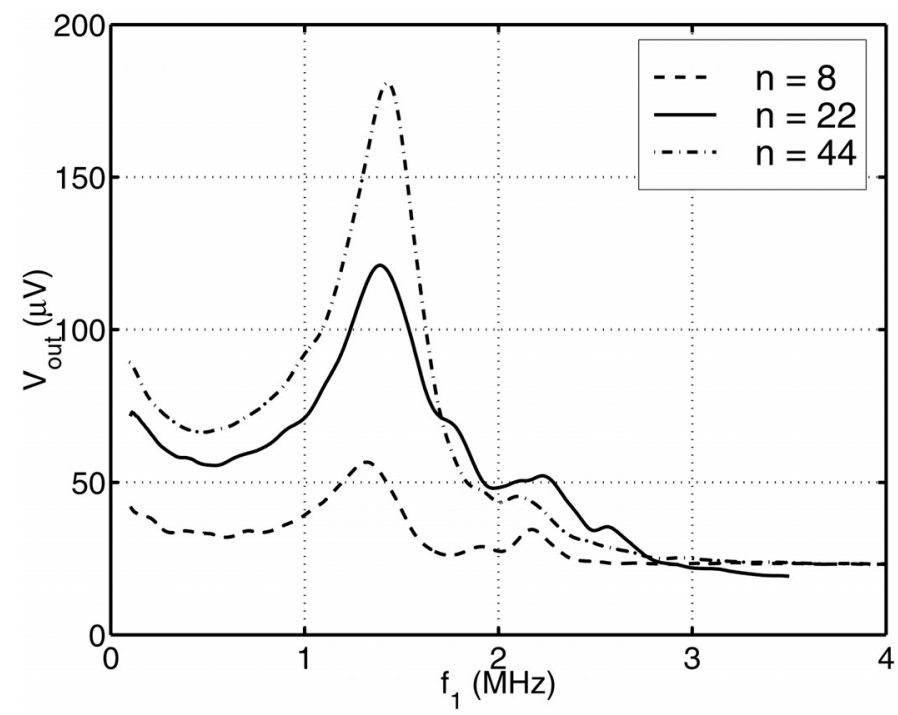

Fig. 12. Excitation experiment results performed on devices with $l_{b}=80 \mu \mathrm{m}$.

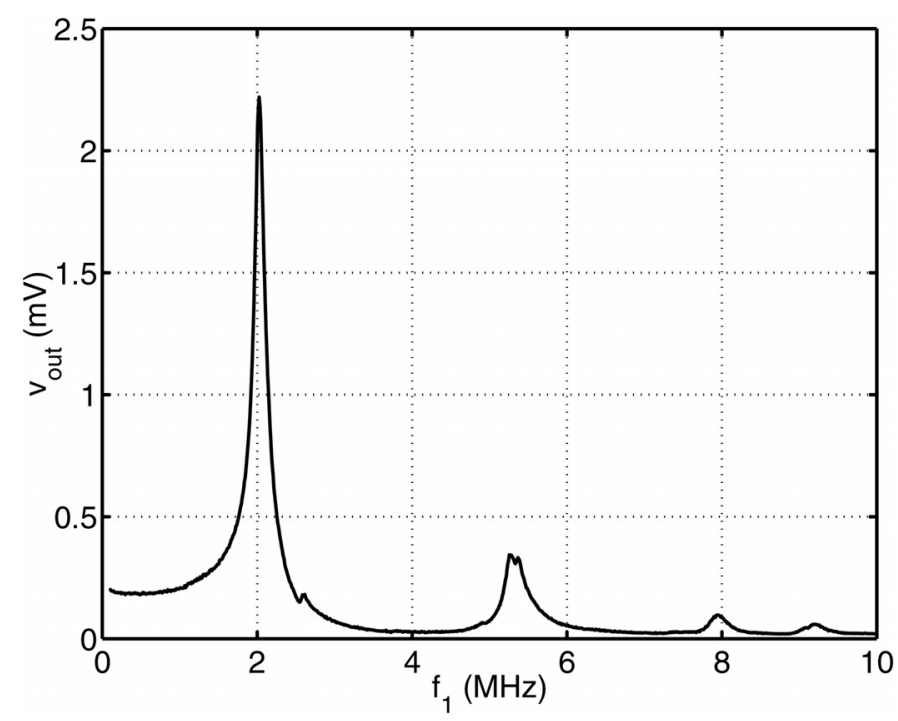

Fig. 13. Excitation experiment results performed on devices with $l_{b}=70 \mu \mathrm{m}$.

amplitude are kept the same. The resonance frequency predicted by (16) for this device is $1.2 \mathrm{MHz}$. However, the first resonance frequency occurs at $2 \mathrm{MHz}$. The percentage shift in the resonance frequency with respect to the predictions is even larger for this device. On the other hand, the Q factor is 13 , which implies lower mechanical loss as compared with the former device. The vibration amplitude is calculated to be $60 \AA / \mathrm{V}$ at the resonance frequency, which again reflects the lower mechanical loss.

The fourth experiment was to place the detector on top of a piezoelectric transducer and drive the piezoelectric transducer with a $10-\mathrm{V}$ AC signal at $2 \mathrm{MHz}$. The wave generated by the transducer travels through the substrate and excites the bridges. The bridge vibrations are measured using our method with direct detection, where a spectrum analyzer is utilized in the same way as the previous ex- 


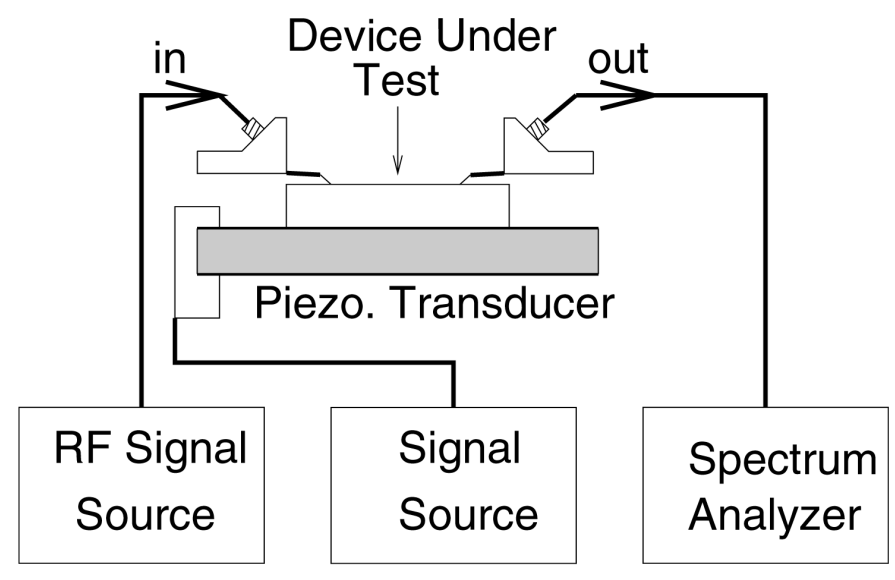

Fig. 14. Detection experiment setup. RF signal source feeds the artificial line, and the signal source drives the piezoelectric transducer. The spectrum analyzer monitors the transmitted signal.

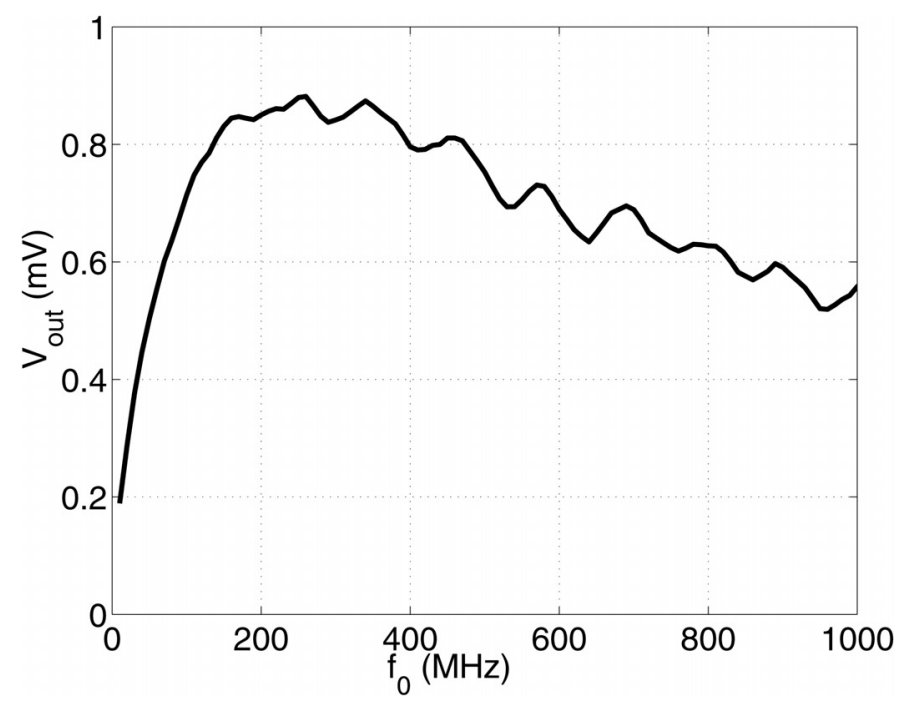

Fig. 15. Si sample. The output power measured as a function of RF signal.

periment. The measurement setup is shown in Fig. 14. We could not make an air-coupled measurement because we could not get close to the detector and the attenuation in air did not allow us to place the transmitter too far. Therefore, we put the transmitter at the back side of the detector. In this way, we were able to couple the ultrasound to our detectors in a more efficient way.

In this experimental setup, we are able to sweep the RF $f_{0}$ while keeping the ultrasound frequency and amplitude constant. In this way, we determined the relative sensitivity as a function of RF. Fig. 15 and 16 show the results of this experiment performed on two different devices with an $\mathrm{RF}$ voltage of $0.33 \mathrm{~V}$. The first one is a device with 22 sections, where the bridge length, width, and thickness are 50, 38, and $1.0 \mu \mathrm{m}$, respectively. The gap thickness was designed to be $1 \mu \mathrm{m}$, but, because of the fabrication process, it turned out to be much lower and very non-uniform. The capacitor dimensions are $38 \mu \mathrm{m}$ on each side, and the inductor width and length are $10 \mu \mathrm{m}$ and $100 \mu$, respec-

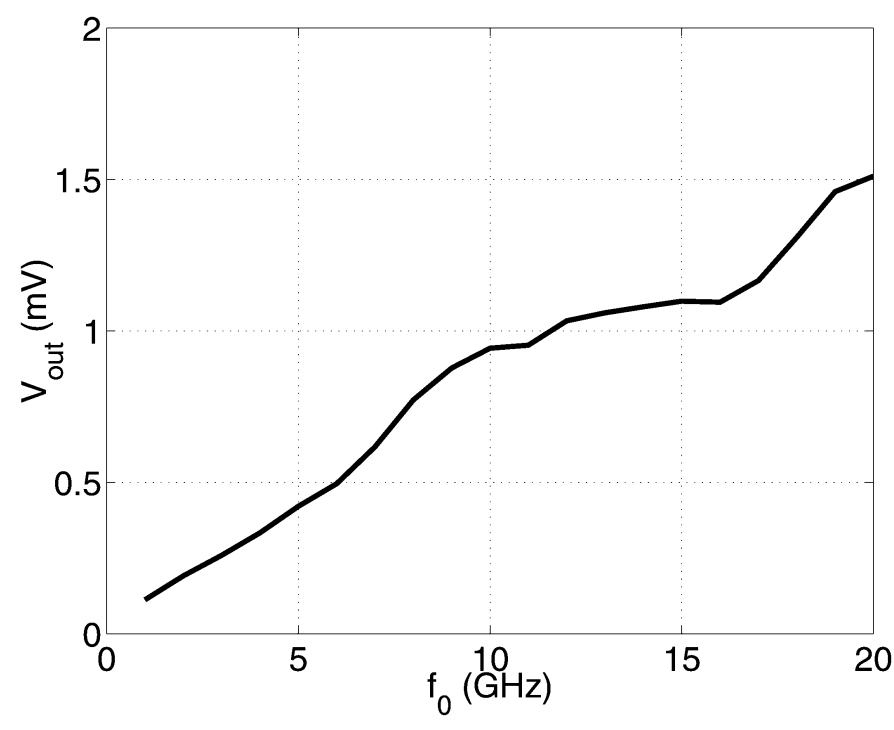

Fig. 16. GaAs sample. The output power measured as a function RF signal.

tively. The bridge material is aluminum, and the substrate is silicon with a resistivity of 3 to $5 \Omega \cdot \mathrm{cm}$. Because of the medium resistivity of the silicon substrate, the RF losses were quite dominant. This limits the maximum $\mathrm{RF}$ we can use to $300 \mathrm{MHz}$ as shown in Fig. 15. However, it is clear from the figure that the sensitivity increases linearly up to the frequency where losses start to dominate.

Fig. 16 shows the results of the same experiment done on the same kind of device built on a semi-insulating GaAs substrate. However, this time the fabrication process was corrected, and the gap thickness obtained was $1 \mu \mathrm{m}$. Using a semi-insulating substrate suppresses the losses considerably and allows us to use quite high RF. The plot shows that the sensitivity does increase with RF, where the optimum frequency for this particular device is $>20 \mathrm{GHz}$. The fluctuations in the sensitivity are caused by the reflections and standing waves in RF path.

In the Mason's equivalent circuit for electrostatic driving systems, the mechanical port is modeled as a series $R_{m} L_{m} C_{m}$ circuit loaded with the acoustical impedance of the surrounding medium. The $R_{m}$ value accounts for the mechanical losses and determines the mechanical bandwidth of the system. The $L_{m}$ and $C_{m}$ values determine the mechanical resonance. At the mechanical resonance frequency of the system, the impedance of $L_{m}$ and $C_{m}$ cancel each other, and the acoustical impedance is real. In the mechanical circuit, the pressure and the particle velocity are analogous to voltage and current of the electrical circuit, respectively.

From excitation experiments, we can measure the vibration amplitudes of the bridges. Because we know the force we apply, it is possible to calculate the acoustical impedance of the bridges at the resonance frequency. The particle velocity is found using $|\dot{x}|=|j \omega x|$. The force we apply is

$$
F_{A C}=\frac{\epsilon_{0} A}{x_{0}^{2}} V_{o f f} V_{1}
$$


TABLE IV

Mason's Equivalent Circuit Values Extracted For the Bridges of the Measured Devices.

\begin{tabular}{ccc}
\hline Device & 1 & 2 \\
\hline$x_{0}(\mu \mathrm{m})$ & 0.35 & 0.4 \\
$V_{\text {out }}(\mathrm{mV})$ & 0.12 & 2.2 \\
$\Delta x(\AA)$ & 6 & 60 \\
$f_{m}(\mathrm{MHz})$ & 1.4 & 2 \\
$\dot{x}(\mathrm{~m} / \mathrm{s})$ & 0.0053 & 0.075 \\
$F_{A C}(\mu \mathrm{N})$ & 0.22 & 0.29 \\
$R_{m}\left(\mathrm{~kg} / \mathrm{m}^{2} \mathrm{~s}\right)$ & $16 \mathrm{k}$ & 250 \\
$Q$ & 4 & 13 \\
$L_{m}\left(\mathrm{~kg} / \mathrm{m}^{2}\right)$ & $7.7 \mathrm{~m}$ & $0.71 \mathrm{~m}$ \\
$C_{m}\left(\mathrm{~m}^{2} \mathrm{~s}^{2} / \mathrm{kg}\right)$ & $1.7 \mathrm{p}$ & $8.9 \mathrm{p}$ \\
\hline
\end{tabular}

where $V_{\text {off }}$ is the offset voltage and $V_{1}$ is the AC voltage amplitude. $A$ is the effective area where the electrostatic attraction takes place. From the force we apply, the pressure on the bridges can be evaluated. Analogous to circuit theory the mechanical loss impedance of the air-bridges is $R_{m}=P /|\dot{x}|-R_{a i r}$ at the resonance frequency, where $R_{\text {air }}$ is the acoustical impedance of air. The $Q$ of the series $R_{m} L_{m} C_{m}$ circuit is

$$
Q=\frac{\sqrt{L_{m} / C_{m}}}{R_{m}}
$$

and the resonance frequency is

$$
f_{m}=\frac{1}{2 \pi \sqrt{L_{m} C_{m}}} .
$$

Because we know $Q_{m}$ and $f_{m}$ from excitation experiments, we can calculate $L_{m}$ and $C_{m}$. For the two devices used in the excitation experiments, we find the mechanical circuit values as shown in Table IV.

The high value of $R_{m}$ calculated for device 1 is because of its low Q. On the other hand, device 2 has a much lower mechanical loss impedance in accordance with the higher $\mathrm{Q}$ value measured. This means that, in transmit, most of the electrical power delivered to the transducer is transmitted into air, and a small portion of it is lost. A similar argument applies on receive too. Because of these reasons, the second device is a good candidate for a pulse-echo measurement, which is our fifth experiment. The result of the experiment is shown in Fig. 17. The device contains 38 sections, which makes a total length of $3.8 \mathrm{~mm}$. The width of the device is $100 \mu \mathrm{m}$. Thus, it is actually a line source in transmitting mode. A cylindrical reflector with a radius of $\sim 1 \mathrm{~cm}$ is placed above the device so that the total distance that the acoustic wave propagates is $\sim 2 \mathrm{~cm}$. The applied pulse is a burst at $2 \mathrm{MHz}$ with a burst count of 40 cycles. The propagation time of the pulse is $\sim 60 \mu \mathrm{s}$, which is also evident from the echo of the transmitted signal.

This experiment is quite important in the sense that the device we used is a very small one and still we are able to detect the echo of the transmitted signal. The received signal is $34 \mathrm{~dB}$ below the transmitted signal; $\sim 13 \mathrm{~dB}$ of it is due to the attenuation of ultrasound in air.

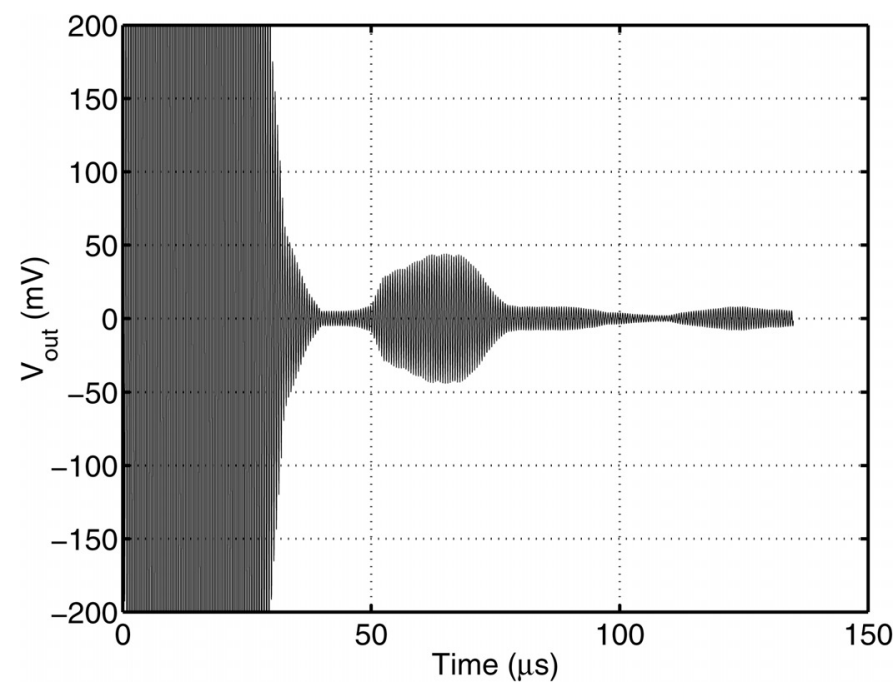

Fig. 17. Pulse-echo measurement result.

\section{CONCLUSION}

In this work, we have introduced a new detection method for CMUTs. The main theme of this new method is the use of a high frequency RF signal instead of a DC bias voltage. There are various advantages of this new method compared with the conventional one. First of all, the sensitivity is improved considerably. The improvement in the sensitivity is determined by the ratio of the RF signal to the ultrasound frequency. Therefore, the higher RF we use, the higher sensitivity we get. Finally, the sensitivity of the new method does not depend on the ultrasound frequency in contrast to the conventional method. Therefore, the improvement in the sensitivity is even better for low frequency applications [24], [25].

The good agreement between the measurements and theory indicate that the model we use and the calculations are quite accurate. Although a spectrum analyzer is used in some of the measurements, the detection circuitry shown in Fig. 3(a and b) can be built on-chip with the transducer resulting in a monolithic system.

For the device on which we performed the DC experiment, the sensitivity is measured to be $\sim 0.2 \mathrm{~V} / \mu \mathrm{m}$. If we compare this sensitivity with thermal noise voltage, we obtain the minimum detectable displacement of the device, which turns out to be $\Delta x_{\min }=\sim 2 \times 10^{-5} \AA / \sqrt{\mathrm{Hz}}$. Recall that the experiment was done at $2 \mathrm{GHz}$ with $1 \mathrm{~V}$ of $\mathrm{RF}$ amplitude. If we increase the RF and amplitude further, reduce the bridge height, and use the optimum number of sections $\Delta x_{\min }$ with an optimized geometry, we can reduce the minimum detectable displacement below $10^{-7} \AA / \sqrt{\mathrm{Hz}}$. However, this is a very rough estimate, which ignores most of the noise sources. An accurate calculation of the minimum detectable displacement requires a thorough noise analysis, which will be done in a separate work. 


\section{REFERENCES}

[1] M. I. Haller and B. T. Khuri-Yakub, "A surface micromachined electrostatic ultrasonic air transducer," in Ultrason. Symp. Proc., pp. 1241-1244, 1994.

[2] I. Ladabaum, B. T. Khuri-Yakub, and D. Spoliansky, "Micromachined ultrasonic transducers: $11.4 \mathrm{mhz}$ transmission in air and more," Appl. Phys. Lett., vol. 68, pp. 7-9, Jan. 1996.

[3] H. T. Soh, I. Ladabaum, A. Atalar, C. F. Quate, and B. T. Khuri-Yakub, "Silicon micromachined ultrasonic immersion transducers," Appl. Phys. Lett., vol. 69, pp. 3674-3676, Dec. 1996.

[4] D. W. Schindel, D. A. Hutchins, L. Zou, and M. Sayer, "The design and characterization of micromachined air-coupled capacitance transducers," IEEE Trans. Ultrason., Ferroelect., Freq. Contr., vol. 42, pp. 42-50, Jan. 1995.

[5] K. Suzuki, K. Higuchi, and H. Tanigawa, "A silicon electrostatic ultrasonic transducer," IEEE Trans. Ultrason., Ferroelect., Freq. Contr., vol. 36, no. 6, pp. 620-627, 1989.

[6] S. Calmes, C. Cheng, F. L. Deg̃ertekin, X. C. Jin, A. S. Ergun, and B. T. Khuri-Yakub, "Highly integrated 2-d capacitive micromachined ultrasonic transducers," in Ultrason. Symp. Proc., pp. 1163-1166, Nov. 1999.

[7] P. Eccardt, K. Niederer, T. Scheiter, and C. Hierold, "Surface micromachined ultrasound transducers in cmos technology," in Ultrason. Symp. Proc., pp. 959-962, 1996.

[8] S. Hansen, N. Irani, F. L. Deg̃ertekin, I. Ladabaum, and B. T. Khuri-Yakub, "Defect imaging by micromachined ultrasonic air transducers," in Ultrason. Symp. Proc., pp. 1003-1006, 1998.

[9] Ö. Oralkan, X. C. Jin, F. L. Deg̃ertekin, and B. T. Khuri-Yakub, "Simulation and experimental characterization of a 2-d capacitive micromachined ultrasonic transducer array element," IEEE Trans. Ultrason., Ferroelect., Freq. Contr., vol. 46, pp. 13371340, Nov. 1999.

[10] - "Simulation and experimental characterization of a 2-d, 3-mhz capacitive micromachined ultrasonic transducer (cmut) array element," in Ultrason. Symp. Proc., pp. 1141-1144, 1999.

[11] A. G. Bashford, D. W. Schindel, and D. A. Hutchins, "Characteristics of ultrasonic micromachined capacitance transducers in water," in Ultrason. Symp. Proc., pp. 955-958, 1996.

[12] B. T. Khuri-Yakub, C.-H. Cheng, F. L. Degertekin, A. S. Ergun, S. Hansen, X.-C. Jin, and O. Oralkan, "Silicon micromachined ultrasonic transducers," Jpn. J. Appl. Phys., vol. 39, pp. 28832887, May 2000.

[13] I. Ladabaum, X.-C. Jin, H. T. Soh, A. Atalar, and B. T. KhuriYakub, "Surface micromachined capacitive ultrasonic transducers," IEEE Trans. Ultrason., Ferroelect., Freq. Contr., vol. 45, pp. 678-690, May 1998.

[14] X.-C. Jin, I. Ladabaum, and B. T. Khuri-Yakub, "The microfabrication of capacitive ultrasonic transducers," J. Microelectromechan. Syst., vol. 7, pp. 295-302, Sep. 1998.

[15] X.-C. Jin, I. Ladabaum, F. L. Deg̃ertekin, S. Calmes, and B. T. Khuri-Yakub, "Fabrication and characterization of surface micromachined capacitive ultrasonic immersion transducers," $J$. Microelectromechan. Syst., vol. 8, pp. 100-114, Mar. 1999.

[16] A. S. Ergun, A. Atalar, B. Temelkuran, and E. Özbay, "A sensitive detection method for capacitive ultrasonic transducers," Appl. Phys. Lett., vol. 72, pp. 2957-2959, Jun. 1998.

[17] — "A new detection method for capacitive micromachined ultrasonic transducers," in Ultrason. Symp. Proc., pp. 1007-1010, 1998.

[18] W. Mason, Electromechanical Transducers and Wave Filters. New York: Van Nostrand, 1942.

[19] A. S. Ergun, "A new detection method for capacitive micromachined ultrasonic transducers," Ph.D. dissertation, Bilkent University, Apr. 1999.

[20] P. M. Morse and K. U. Ingard, Theoretical Acoustics. Princeton, NJ: Princeton University Press, 1968.

[21] R.W.P. King, Transmission-Line Theory. New York: McGrawHill Book Company, Inc., 1955.

[22] C. Goldsmith, J. Randall, S. Eshelman, T. H. Lin, D. Denniston, S. Chen, and B. Norvell, "Characteristics of micromachined switches at microwave frequencies," MTT-S Digest, pp. 1141$1144,1996$.

[23] N. S. Barker and G. M. Rebeiz, "Distributed mems true-time delay phase shifters and wideband switches," IEEE Trans. Microwave Theory Tech., vol. 46, pp. 1881-1890, Nov. 1998.
[24] H. Dudaicevs, M. Kandler, Y. Manoli, W. Mokwa, and E. Spiegel, "Surface micromachined pressure sensors with integrated cmos read-out electronics," Sens. Actuators A, vol. 43, no. 2, pp. 157-163, 1994.

[25] Y. S. Lee and K. D. Wise, "A batch-fabricated silicon capacitive pressure transducer with low temperature sensitivity," IEEE Trans. Electron. Devices, vol. 29, pp. 42-48, Jan. 1982.

A. Sanlı Ergun (S'96-A'98) was born in Ankara, Turkey in 1969. He received his B.Sc., M.Sc., and Ph.D. degrees in 1991, 1994, and 1999, respectively, all in electrical and electronics engineering, from Bilkent University, Turkey.

He was a research assistant in Bilkent University, Turkey between 1991 and 1999. He is now in E.L. Ginzton Laboratory, Stanford University as an engineering research associate. His research interests are microwave electronics, ultrasonics, MEMS, and, specifically, cMUTs.

He is a member of the IEEE and the Electron Devices Society.

Burak Temelkuran was born Izmir, Turkey in 1971. He received his B.S., M.S., and Ph.D. degrees in 1993, 1996, and 2000, respectively, all in physics, at Bilkent University of Turkey. He was a research assistant in Bilkent University, Turkey from 1993 to 2000.

Currently, he is working in the Research Laboratory of Electronics and Department of Materials Science and Engineering, Massachusetts Institute of Technology as a postdoctoral associate.

His research interests are physics, application and fabrication of photonic crystals, fabrication of ultrasonic transducers, and silicon micromachining techniques.

He is a member of American Physical Society and Optical Society of America. He received the New Focus Student Award at the 1999 OSA Annual Meeting.

Ekmel Özbay (M'98) was born on March 25, 1966 in Ankara, Turkey. He received his B.S. degree in electrical engineering from the Middle East Technical University, Ankara, Turkey in 1983. He received his M.S. and Ph.D. degrees from Stanford University in electrical engineering in 1989 and 1992, respectively. During his thesis work, he focused on high speed resonant tunneling and optoelectronic devices. From 1992 to 1994, he worked as a scientist in DOE Ames National Laboratory in Iowa State University in the area of photonic band gap materials. He joined the faculty of physics department of Bilkent University (Ankara, Turkey) in December 1994, where he is currently an associate professor. His research in Bilkent involves photonic crystals, silicon micromachining, and high speed optoelectronics. He is the 1997 recipient of the Adolph Lomb Medal of Optical Society of America. He has authored or co-authored more than 80 articles in scientific journals, conference proceedings, and books.

Abdullah Atalar (M'88-SM'90) was born in Gaziantep, Turkey. He received his B.S. degree from the Middle East Technical University in 1974 and his M.S. and Ph.D. degrees from Stanford University in 1976 and 1978, respectively, all in electrical engineering. From 1978 to 1980 , he was first a post doctoral fellow and later an engineering research associate at Stanford University. From 1980 to 1986, he was on the faculty of the Middle East Technical University as an assistant professor. In 1986, he joined the Electrical and Electronics Engineering Department of Bilkent University and served in the founding of the Department where he is now a professor. In 1996, he was in the Ginzton Lab of Stanford University on a sabbatical leave. Since 1997, he has been the Provost of Bilkent University. 